\title{
The Performance of AODV Routing Protocol Based On Dropped Packets and Throughput Metrics: A Simulation and Comparative Study for VANET
}

\author{
Mazlan Osman \\ Faculty of Computer and Mathematical Science \\ Universiti Teknologi MARA (UiTM) \\ Terengganu, Malaysia \\ mazlanos@tganu.uitm.edu.my
}

\begin{abstract}
Vehicular Ad hoc Network (VANET) is a challenging network environment in which communication between vehicles in highly fading environments, like an urban scenario, is unpredictable and difficult. In order to analyze the performance of routing protocols and applications, a few of network simulators could be applied such as GlomoSim, NS-2, OPNET, QualNet and NCTUns. In this dissertation, we study either hindering object and signal strength in VANET could affected the performance of AODV routing protocol or not and what are the other factors affect AODV performance. The performance analysis of AODV routing protocols under hindering object and signal attenuator is presented via simulation using NCTUns network simulator based on dropped packet and throughput metrics. Beside simulation, this paper also presented comparative analysis for AODV measured by throughput and end-to-end delay. Based on simulation process and comparative analysis, it is observed that the performance of AODV is affected by physical hindering objects and signal strength. It is also observed that the performance of AODV is independent from the types of network simulator used in simulation process.
\end{abstract}

\section{Indexing terms/Keywords}

Routing Protocol, VANET, Simulation

\section{Academic Discipline And Sub-Disciplines}

Computer Science

\section{SUBJECT CLASSIFICATION}

Computer Networking

\section{TYPE (METHOD/APPROACH)}

Documents Review and Simulation Study

\section{Council for Innovative Research}

Peer Review Research Publishing System

Journal: International Journal of Management \& Information Technology

Vol.4, No.2

editor@cirworld.com

www.cirworld.com, member.cirworld.com 


\section{INTRODUCTION}

\subsection{Background}

Today, the main problem in the road traffic, especially in a big city, is traffic congestion. The congestion and related vehicle accommodation problem is accompanied by a constant threat of accidents as well. Absence of road traffic safety takes a toll of precious human lives and poses a dire threat to our environment as well. It also causes the waste of energy and environmental pollution.

Preliminary precautions such as seat belts and airbags are used but it cannot eliminate problems due to driver's inability to foresee the situation ahead of time [8]. On a highway, a problem arises if a vehicle cannot currently predict the speed of other vehicles. However, with the use of sensor, computer and wireless communication equipment, speed could be predicted and a warning message could be sent to limit the risk of potential accidents [3].

A routing protocol governs the way that two communication entities exchange information that includes the procedure in establishing a route, decision in forwarding and action in maintaining the route or recovering from routing failure. Nowadays, there are a lot of numbers of routing protocols exist for VANET. Some of them are designed to handle a special condition or a special problem. Despite the special condition or problem that these routing protocols are considering or addressing, there is no agreed-upon standard or benchmark to validate their performance. The benchmark not only includes a standard routing protocol but also a simulation environment. The open issue in VANET routing protocol is then whether there is any benchmark tool for evaluating these protocols.

\subsection{Ad-Hoc On Demand Distance Vector - AODV}

The Ad hoc On Demand Distance Vector (AODV) routing algorithm is a routing protocol designed for ad hoc mobile networks. AODV is capable of both unicast and multicast routing. It is an on demand algorithm, meaning that it builds routes between nodes only as desired by source nodes. It maintains these routes as long as they are needed by the sources. Additionally, AODV forms trees which connect multicast group members. The trees are composed of the group members and the nodes needed to connect the members. AODV uses sequence numbers to ensure the freshness of routes. It is loop-free, self-starting, and scales to large numbers of mobile nodes.

AODV builds routes using a route request / route reply query cycle. When a source node desires a route to a destination for which it does not already have a route, it broadcasts a route request (RREQ) packet across the network. Nodes receiving this packet update their information for the source node and set up backwards pointers to the source node in the route tables. In addition to the source node's IP address, current sequence number, and broadcast ID, the RREQ also contains the most recent sequence number for the destination of which the source node is aware. A node receiving the RREQ may send a route reply (RREP) if it is either the destination or if it has a route to the destination with corresponding sequence number greater than or equal to that contained in the RREQ. If this is the case, it unicasts a RREP back to the source. Otherwise, it rebroadcasts the RREQ.

Nodes keep track of the RREQ's source IP address and broadcast ID. If they receive a RREQ which they have already processed, they discard the RREQ and do not forward it. As the RREP propagate back to the source, nodes set up forward pointers to the destination. Once the source node receives the RREP, it may begin to forward data packets to the destination. If the source later receives a RREP containing a greater sequence number or contains the same sequence number with a smaller hop count, it may update its routing information for that destination and begin using the better route. As long as the route remains active, it will continue to be maintained. A route is considered active as long as there are data packets periodically traveling from the source to the destination along that path. Once the source stops sending data packets, the links will time out and eventually be deleted from the intermediate node routing tables. If a link break occurs while the route is active, the node upstream of the break propagates a route error (RERR) message to the source node to inform it of the now unreachable destination(s). After receiving the RERR, if the source node still desires the route, it can reinitiate route discovery.

The advantage with AODV compared to classical routing protocols like distance vector and link-state is that AODV is greatly reduced the number message in the network. AODV achieves this by using a reactive approach. This is probably necessary in an ad-hoc network to get reasonably by performance when the topology is changing often.

AODV is also routing in the more traditional sense compared to for instance source routing based proposals like DSR. The advantage with a more traditional routing protocol in an ad-hoc network is that connections from the ad-hoc network to wired network like the Internet is most likely easier.

AODV only support one route for each destination. It should however be fairly easy to modify AODV, so that it supports several routes per destination. Instead of requesting a new route when an old route becomes invalid, the next stored route to that destination could be tried. The probability for that route to still be valid should be rather high.

AODV uses hello messages at the IP-level. This means that AODV does not need support from the link layer to work properly. It is however questionable if this kind of protocol can operate with good performance without support from the link layer.

We can consider many situations to perform a VANET simulation using AODV and we can simulate a bundle of possibilities in an urban environment especially to evaluate the performance when something happens in the surrounding area. What happens, for instances, when hindering objects between communicating vehicles exist? When two vehicles communicating each other across different lanes and encountered by obstacle like building, will they be able to continue their communication? How will they maintain the communication between nodes? Moreover, what happens when the strength of signal changes during communication occur between nodes. 


\section{LITERATURE REVIEW}

\section{Vehicular Ad Hoc Networks (VANETS)}

At present, VANET is a widely discussed area of wireless communication. As we know, VANET is a type of MANET where nodes represents vehicles moving at high pace and vehicle traffic determined regularity. This technology enables communication between vehicles and nearby road-side infrastructure and is made possible through a wireless sensing device installed in the vehicles. With the inception of VANET, new opportunities and related technologies such as applications for traffic jam, accident control and weather updates have appeared.

The architecture of VANETs falls within three categories: pure cellular/WLAN, pure ad hoc, and hybrid. In pure cellular/WLAN architecture, the network uses cellular gateways and WLAN access points to connect to the Internet and facilitate vehicular applications. Vehicles communicate with the Internet by driving by either a cellular tower or a wireless access point.

Since the infrastructure of cellular towers and wireless access points are not necessarily widely deployed due to costs or geographic limitations, nodes may only engage in communication with each other. Information collected from sensors on a vehicle can become valuable in notifying other vehicles about traffic condition and helping the police solve crimes [30]. The infrastructure-less network architecture is in the pure ad hoc category where nodes perform vehicle-to-vehicle communication with each other.

When there are roadside communication units such as a cellular tower and an access point and vehicles are equipped with wireless networking devices, vehicles can take advantage of the infrastructure in communicating with each other. Various applications in areas of urban monitoring, safety, driving assistance, and entertainment [12] have used infrastructure communicating units to access dynamic and rich information outside their network context and share this information in a peer-to-peer fashion through ad hoc, infrastructure-less communication. The hybrid architecture of cellular/WLAN and ad hoc approaches provides richer contents and greater flexibility in content sharing.

Similar to mobile ad hoc networks (MANETs), nodes in VANETs self-organize and self-manage information in a distributed fashion without a centralized authority or a server dictating the communication. In this type of network, nodes engage themselves as servers and/or clients, thereby exchanging and sharing information like peers. Moreover, nodes are mobile, thus making data transmission less reliable and suboptimal.

Even though the performance can be tested in real situations but some factors must be think in depth like cost, inaccurate results and protocol evaluation of complex environment. An automated tool called network simulator can imitate the protocol and produce a similar result to the real world. VANET and MANET is wireless ad hoc network but it differ each other. In VANET, the nodes strictly follow the traffic rules and their kind of movement is very complex. It is important to generate a realistic mobility model that is as realistic as real ad-hoc network communication if we want to obtain the good results from VANET simulation. The usage of mobility model signifies the movement of mobile node that will consume the routing protocol.

\section{VANET Routing Procotols}

Generally, a routing protocol governs the way that two communication entities exchange information; it includes the procedure in establishing a route, decision in forwarding, and action in maintaining the route or recovering from routing failure. The routing protocol has two main functions. First, select the routes for various source-destination pairs and second, deliver the message to their correct destination.

This section will describe several types of VANET routing protocols exist today. The following are some of the routing protocols could be used for VANET.

\section{AODV (Ad Hoc On Demand Vector)}

In AODV [16] routing, nodes record the address of the node sending the query in their routing table upon receipt of a broadcast query (RREQ). This procedure of recording its previous hop is called backward learning. Upon arriving at the destination, a reply packet (RREP) is then sent through the path, the node obtained from backward learning to the source. At each stop of the path, the node would record its previous hop, thus establishing the forward path from the source. After the path has been established, it is maintained as long as the source uses it. A link will be reported recursively to the source and will in turn trigger another query-response procedure to find a new route. AODV is topology-based routing protocol.

\section{AODV+PGB (Preferred Group Broadcasting)}

AODV+Preferred Group Broadcasting (PGB) is a broadcasting mechanism that aims to reduce broadcast overhead associated with AODV's route discovery and to provide route stability especially important in VANETs where fast moving vehicles are used as wireless hosts. Based on the received signal of the broadcast, receivers can determine whether they are in the preferred group and which one in the group to broadcast. Since only one node is allowed to broadcast and since the preferred group is not necessarily the one that makes the most progress towards the destination, route discovery might take longer than before. Another drawback is that broadcast can discontinue if the group is found to be empty (possibly because of sparse networks). Packet duplication can happen as two nodes in the preferred group can broadcast at the same time. According to Naumov et al. [26], the way to deal with broadcast duplication is to add packet's predecessors into the packet. This creates the same type of overhead in the packet as DSR.

\section{DSR (Dynamic Source Routing)}


DSR is another type of topology-based routing protocol. In DSR, the source indicates in a data packet's sequence of intermediate nodes that it has traversed. The destination then retrieves the whole path from the query packet and uses it to respond the source. As a result, the source can establish a path to the destination. If the destination is allowed to send multiple routes replies, the source node may receive and store multiple routes from destination. When some link in the current route breaks, an alternative route could be used. DSR is better used in a network with low mobility.

\section{TORA (Temporally Ordered Routing Algorithm)}

In TORA [17], a directed acyclic graph (DAG) toward the destination is built based on the height of the tree rooted at the source. The directed acyclic graph directs the flow of packets and ensures reachability to all nodes. When a node has a packet to send, it broadcasts the packet. Its neighbour only broadcasts the packet if it is the sending node's downward link based on the DAG. A node would construct the directed graph by broadcasting a query packet. Upon receiving a query packet, if a node has a download link to the destination, it will broadcast a reply packet, otherwise it simply drops the packet. Upon receiving a reply packet, a node will update its height only if the height from the reply packet gives the minimum of all the heights from reply packets it has received. Then it broadcast the reply packet.

\section{NCTUns Simulator}

The best way to obtain the network's performance is through simulation. There are two factors should be considered in VANET simulation. First, the traffic simulation and the second is network simulation. The traffic simulation assists in creating traces of urban mobility model and this information is fed into network simulation. The network simulation creates topologies between the nodes and vice versa. A number of simulations exist for VANET but none of them have been up to the mark and none of them can provide a completion solution for simulating VANET. This part will explores one of the simulator called NCTUns.

NCTUns (National Chiao University Network Simulator) is based on Harvard simulator. It was proposed by S. Y. Wang in 2002. NCTUns is purely written in C++ with a powerful GUI support. The user need not to worry about the complex coding because NCTUns hides the complexities with a few mouse clicks. Since its first released, NCTUns has supported a distributed simulation approach to run simulation tasks concurrently. Furthermore, NCTUns also can simulate $802.11 \mathrm{a}$ $802.11 \mathrm{~b}$ and $802.11 \mathrm{~g}$ technologies. It includes free space, two ray ground and free space with a shadowing path loss model. It moreover includes Rayleigh and Ricean as a fading model. NCTUns implements directional, bidirectional and rotating antenna types. NCTUns implements block objects to introduce the hindering object between wireless signals. The wall object can completely block the wireless signal or can attenuate the signal with the specified value. The hindering object gives good simulation environment to observe the effects of multihop wireless network simulation. During simulation, each node is allowed to send either a UDP or multiple TCP/IP packets.

\section{RESEARCH METHODOLOGY}

\section{Type of Methodology}

There are various methods in collecting information or more precisely data gathering. It can be carried out by document reviews, searching the related topic literatures, white papers, technical papers, marketing reports, conferences proceedings, product data sheets, product brochures, Internet and web pages, project focus groups, conducting interviews with experienced personnel, distributing questionnaire, or even communicate to people.

The methods used for this research are the review of literatures, journals and books from the Internet and Web pages and also by simulation process. It is important to know the right method during handling the dissertation or thesis project in order to get the good data and result that suit to the objective.

\section{Document Reviews}

There are so many documents could be reviewed for obtaining data especially about VANET. Document reviews could be reviewing the electronic documents or printed documents, for example, reviewing documents and books, reports and journals from the Internet and Web pages or other pieces of written information for content and themes. The reviewing process is where and when the sticky notes were placed, written on or even attached to other reading materials to the copies that were read to make the document reviews more comprehensive and easier for references. All these are useful sources for the content of this thesis. Those documents or reports are published based on the study of current markets and trends. The findings or the results from this method can be easily quantified and analyzed later. The findings obtained from this method will be evaluated, analyzed and compared descriptively.

\section{Simulation Study}

The second method used in this thesis is simulation, means that, related to the thesis objective, we had study the simulation process for VANET using one of the selected VANET network simulator exist today.

One of the primary advantages of simulation is it enables to provide users with practical feedback when designing real world systems. This allows the designer to determine the correctness and efficiency of a design before the system is actually constructed. Consequently, the user may explore the merits of alternative designs without actually physically building the systems. By investigating the effects of specific design decisions during the design phase rather than the construction phase, the overall cost of building the system diminishes significantly.

\section{Data Analysis}




\section{Performance Evaluation}

In order to measure the performance of VANET simulation, we used two types of metrics that describe the characteristics and behaviour of VANET, especially the communication between two nodes through obstacles or hindering object and through signal attenuator with different strength of $\mathrm{dbm}$. The two related metrics are throughput and dropped packet.

There performance of simulation will be analyzed and evaluated using both metrics and the relationship between these two metrics will be shown in a graph.

\section{Comparative Analysis}

As mentioned previously, the findings obtained from the selected articles and journals will be evaluated, analyzed and compared descriptively. The metrics used for evaluation and comparison are packet delivery fraction (equivalent to throughput) and end-to-end delay (equivalent to dropped packet).

\section{Simulation Setup}

In the previous chapter, we have discussed various types of simulation tools for performing realistic VANET simulation. Based on the survey, we had selected NCTUns 5.0 simulator because of its added features. NCTUns requires Ubuntu or Fedora with GCC version 4.3 or above. The scenario presents a simple topology with six vehicles communicating with each other.

Each vehicle is equipped with 802.11a wireless module using CSMA/CD MAC protocol. The length of the road is set to more that 1000 meters. The distance between each vehicle on the same road is set to 60 meters, whereas the distance between each vehicle on the opposite road is set to about 238 meters. The transmission range of each vehicle module is 300 meters. Each vehicle moves at a speed of $10 \mathrm{~m} / \mathrm{s}$. Of course, the routing protocol used is AODV. The path loss model (attenuation) used here is free space and showing using Rayleigh fading (magnitude of signals through communication medium will vary randomly).

\section{RESEACRH ANALYSIS}

This chapter consists of two sections. First, the simulation study of AODV routing protocols when hindering objects and signal attenuator exists, and secondly, the comparative study of AODV routing protocol for the different types of network simulators.

\section{Simulation Study}

When the simulation starts, node 3 communicates with node 6 in a free medium with no external stimulus. The medium was free up until 3.0 seconds. After 3.0 seconds, node 2 started to communicate with node 7 using the same medium. At 5.0 seconds, node 4 started to communicate with node 5 .

The whole simulation runs for 80 seconds and within these 80 seconds, all nodes encounter some kind of disturbance in one form or the other. All the following graphs captured the events as they occurred.

The metric used to evaluate the performance are throughput and dropped packet. Throughput is the average rate of successful message delivery over communication channel. This data may be delivered over a physical or logical link, or pass through a certain network node. Dropped packet is a type of Quality of Service (QoS) whereas the packet drops occur when a queue within a network router reaches its maximum length. When a packet drop occurs, connection-based protocols such as TCP slow down their transmission rates in an attempt to let queue packets be serviced.

\section{Communication without Obstacles (Metric Dropped Packet)}

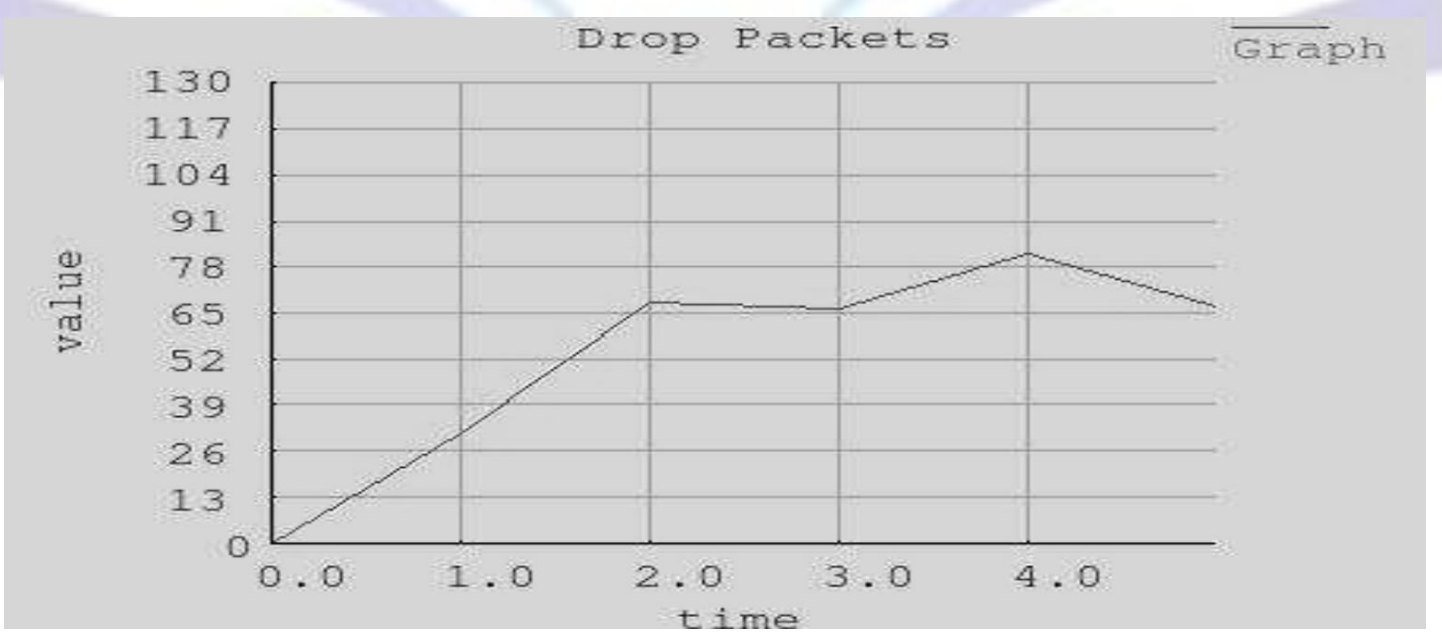

Fig. 1. Communication without Obstacles (Dropped Packet) 
From the above graph, we can see that the dropped packet increased when the node 3 and node 6 communicating each other. It occurs for 2 seconds. It means that in the first 2 seconds in node communication, the performance of data transmission is good. After two seconds, when a queue reaches at maximum length, the dropped packet is decreased and this situation occurs in 1 second. After 3 seconds, the medium was free up.

After that, node 2 starts communicate with node 7 . The communication occurs in 2 seconds. From the above graph, it shows that the dropped packet is increased again for 1 minute. After that, when a queue reaches at maximum length, the dropped packet is decreased and this situation occurs in 1 second.

\section{Communication without Obstacles (Metric Throughput)}

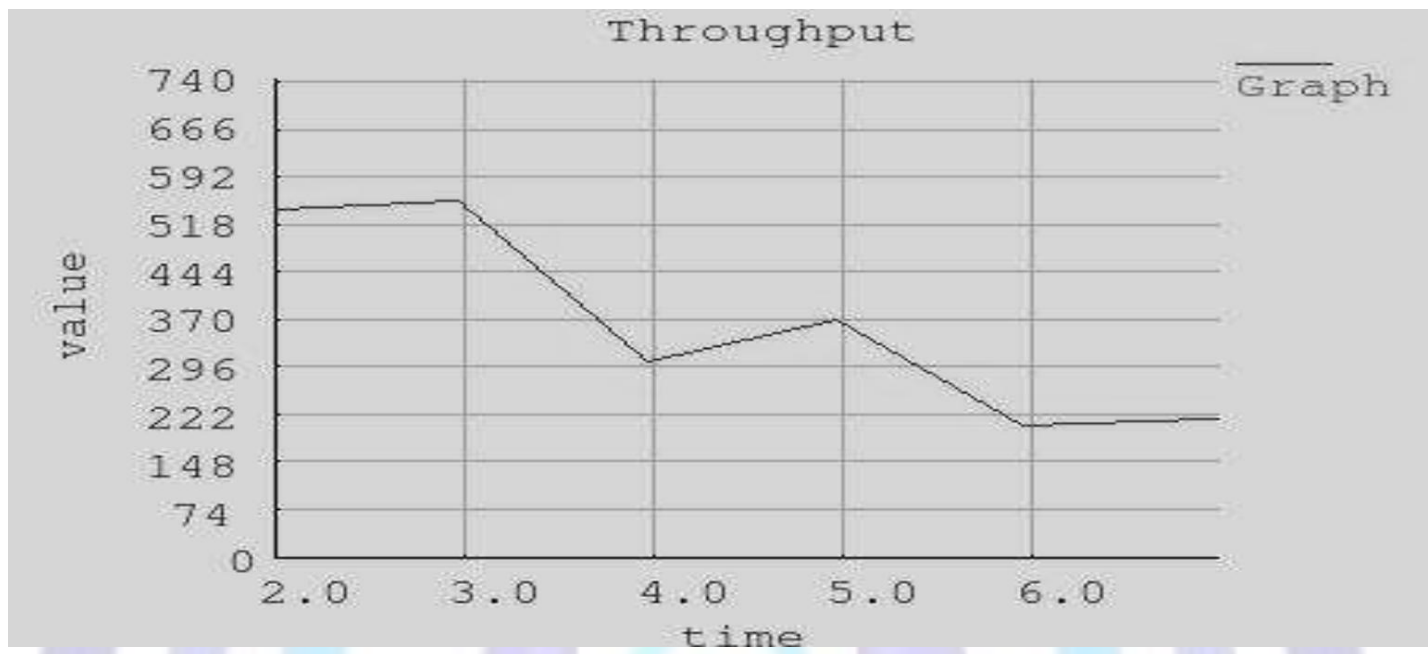

Fig. 2. Communication without Obstacles (Throughput)

Based on the above graph (Fig. 2), it shows that the average of successful data delivery or throughput is also increased when node 3 communicate with node 6 . But the graph is slightly difference after 2 seconds compared to the previous graph (Figure 4.1). In the above graph, after 2 seconds, the throughput is still increased even though the dropped packet decreased. After 3 seconds, when node 2 starts communicate with node 7, the throughput is decreased for 1 minute. But when we compared to the previous graph (Figure 4.1), the dropped packet increased when the communication started. After that, when a queue reaches at maximum length, the dropped packet is decreased back and this situation occurs in 1 second. At 5 seconds, node 4 starts communicate with node 5 and the same phenomena occur during communication in term of the relationship between the dropped packet and throughput.

\section{Communication with Obstacles (Metric Dropped Packet)}

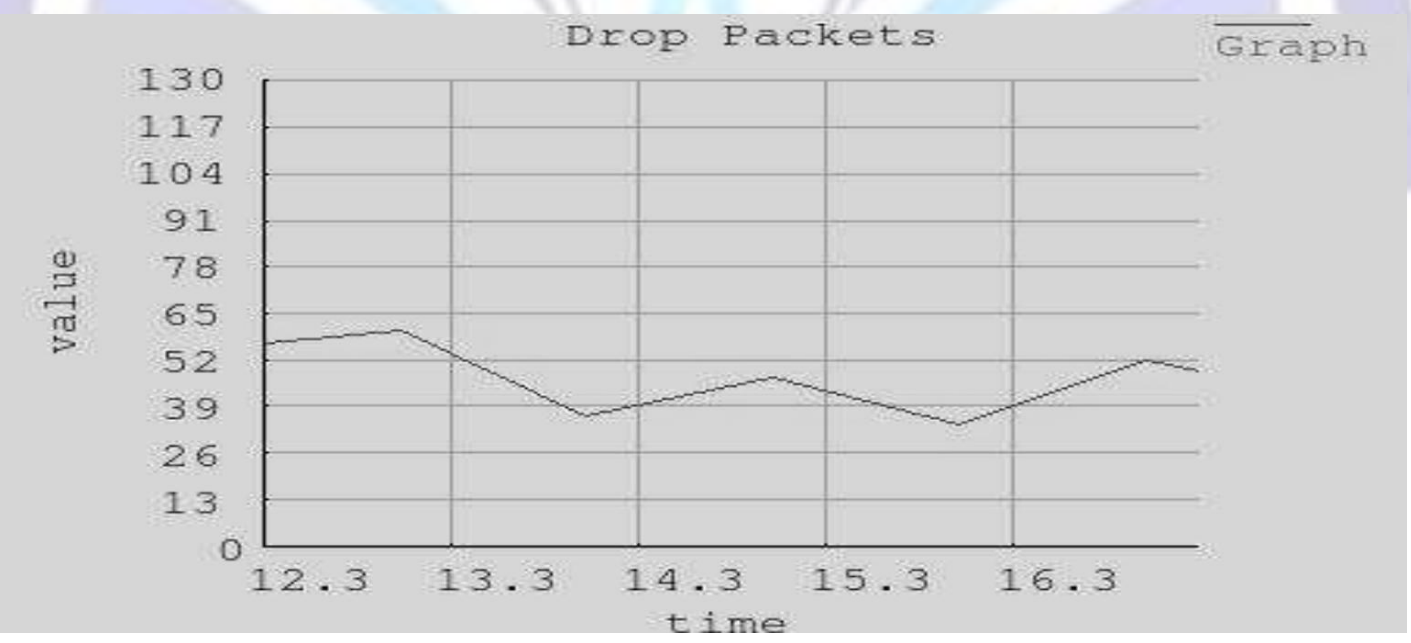

Fig.3. Communication with Obstacles (Dropped Packet)

After 14.3 seconds, the signal strikes some object and some proportion of signal was scattered in the other direction. The duration is about in 8 seconds. The above graph (Fig. 3) show the performance of dropped packets when obstacle appears during communication. From the graph, we can see that the dropped packet is decreased and increased for 2 seconds when the nodes facing an obstacle in their communication. But after 2 seconds, the dropped packet in decreased gradually. 


\section{Communication with Obstacles (Metric Throughput)}

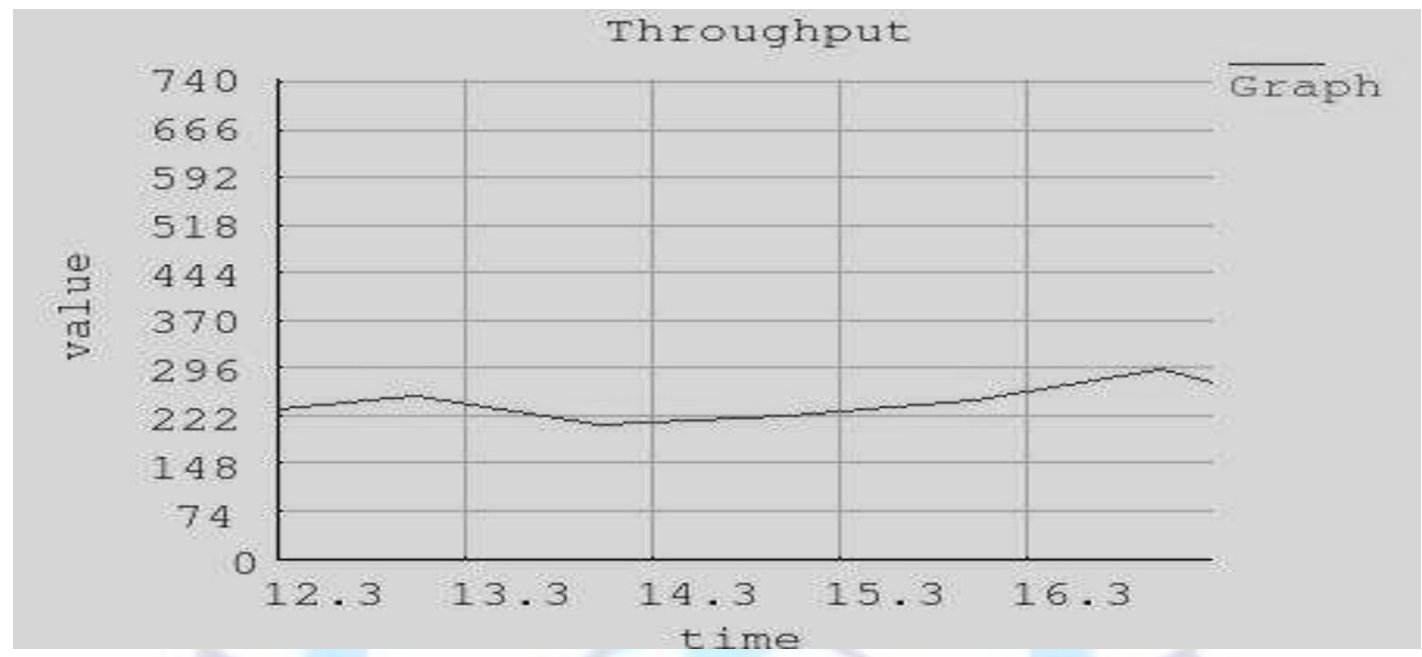

Fig.4. Communication with Obstacles (Throughput)

The above graph (Fig. 4) show the performance of throughput when obstacle appears during communication. From the graph, we can see that the throughput is increased in 2 seconds when the nodes facing an obstacle in their communication. But after 2 seconds, the same thing happen as occur to dropped packet whereas the throughput started decreased gradually.

\section{Multi-hoping (Metric Dropped Packet)}

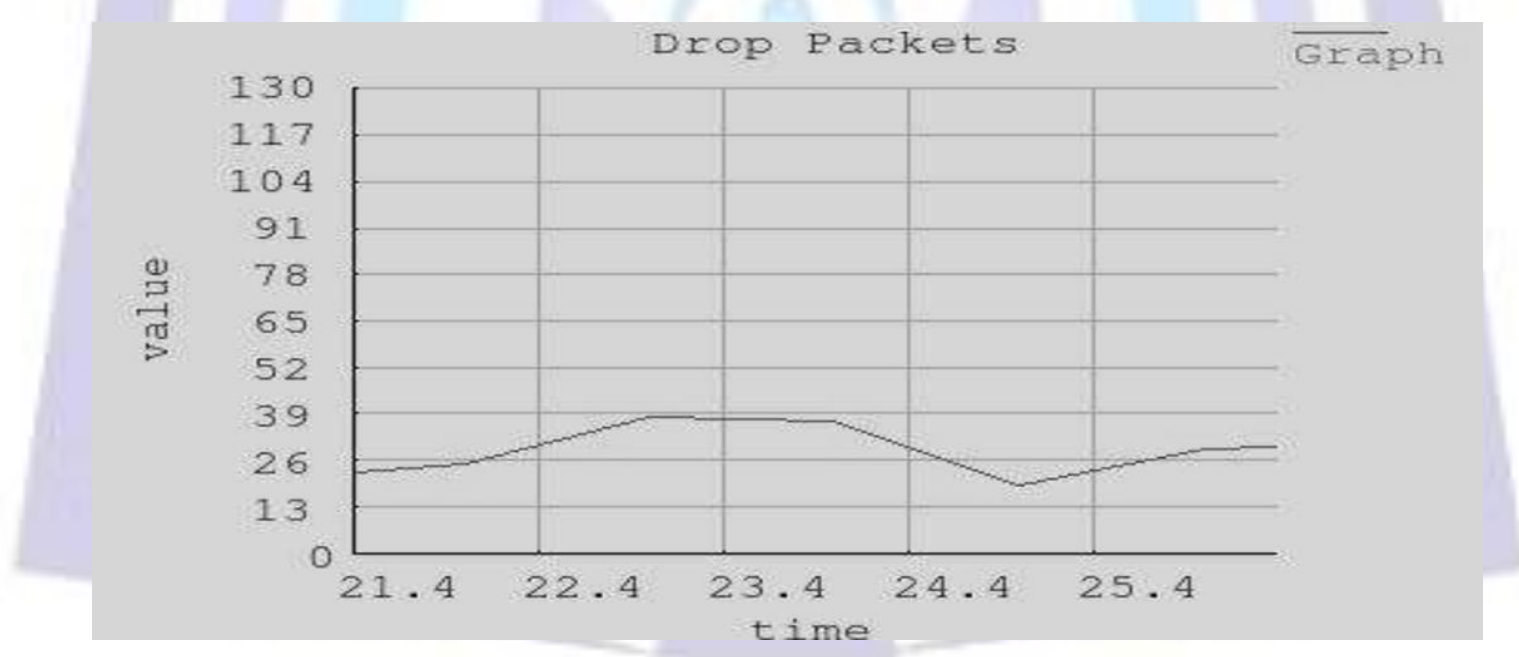

Fig. 5. Multi-hoping (Dropped Packet)

After 8 seconds communication via obstacles, it means that at 21.4 seconds, the communication between node 2 and node 6 was blocked. To communicate with node 6 , node 2 was using node 4 and node 5 as a communication medium to reach to node 6. The dropped packet is shown in the above graph (Figure 4.5) when using two hops for communication.

As mentioned before the dropped packet gradually decreased during communication with obstacles. We can see this situation in the above graph whereas the last value of dropped packet before striking the object is about 39 packet/s but after 8 seconds, the value of dropped packet is below than 25 packet/s.

\section{Multi-hoping (Metric Throughput)}

The throughput after 8 seconds communication via obstacles is shown in the above graph (Fig. 6). As mentioned in the previous section, at 21.4 seconds, the communication between node 2 and node 6 was blocked and to communicate with node 6 , node 2 was using node 4 and node 5 as a communication medium to reach to node 6 . It means that it using two hops for communication. 


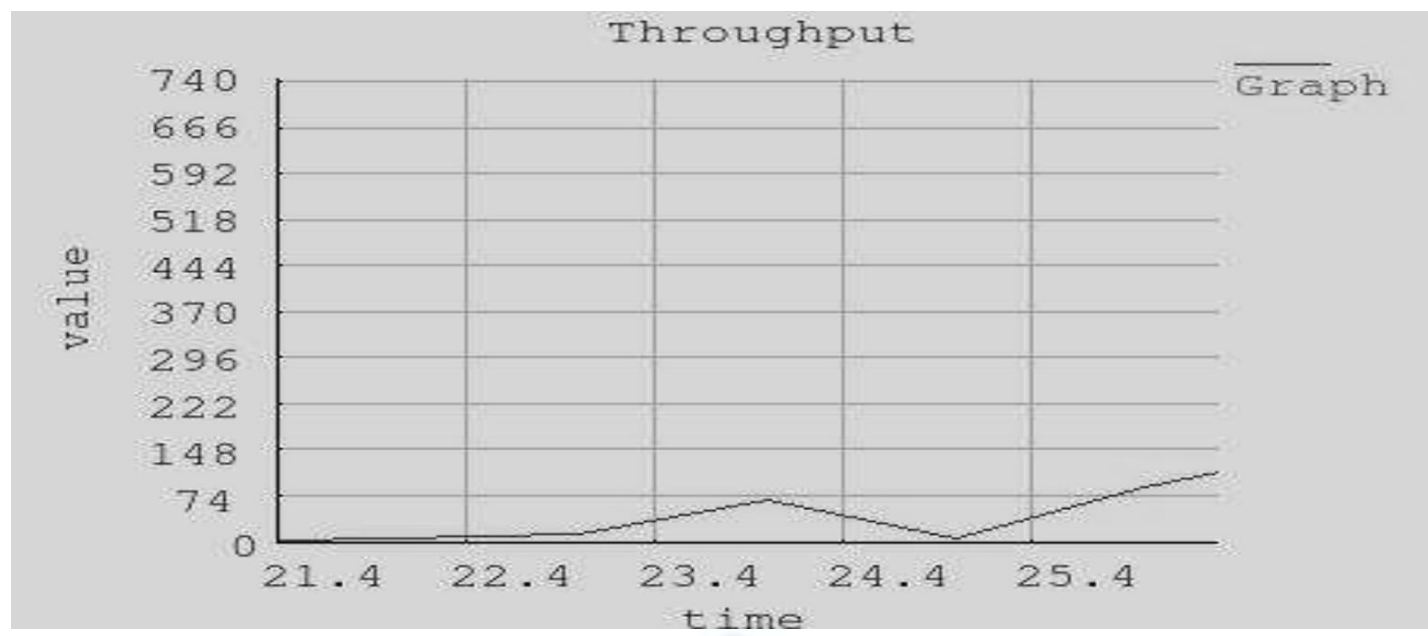

Fig. 6. Multi-hoping (Throughput)

The throughput gradually decreased during communication with obstacles as explained before. The above graph had approved this situation whereas the last value of throughput before striking the object is about 222 bps (Figure 4.4) but after 8 seconds, the value of throughput is going to zero.

\section{Signal attenuated by $2 \mathrm{dbm}$ (Metric Dropped Packet)}

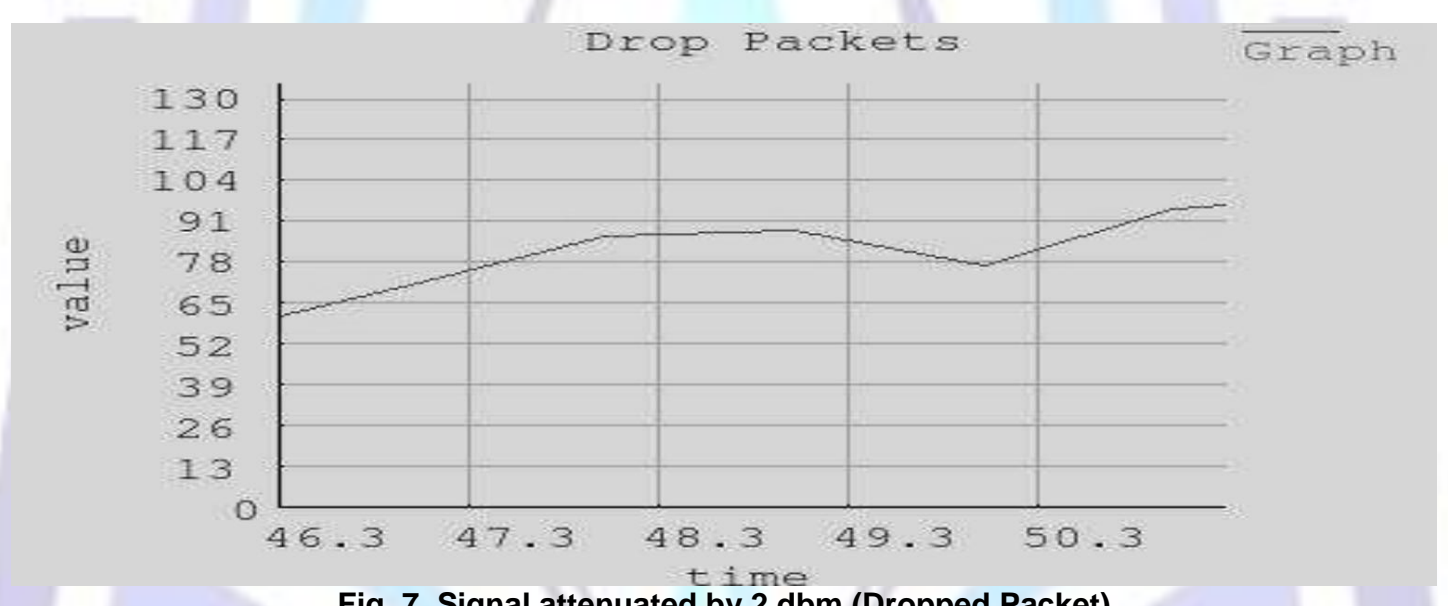

Fig. 7. Signal attenuated by $2 \mathrm{dbm}$ (Dropped Packet)

After exists from obstacles, the communication continues as normal for 24 seconds. At 46.3 seconds, the node reached 2 $\mathrm{dbm}$ signal attenuator during communication.

At this time, the signal strength is attenuated by $2 \mathrm{dbm}$ (free medium). This situation occurs within 7 seconds. The above graph show the dropped packet when the signal strength is attenuated by $2 \mathrm{dbm}$. As a result shown in the above graph (Fig. 7), starting from 46.3 seconds, the number of dropped packets is increased from about 62 packet/s to the above 90 packet/s.

\section{Signal attenuated by $2 \mathrm{dbm}$ (Metric Throughput)}

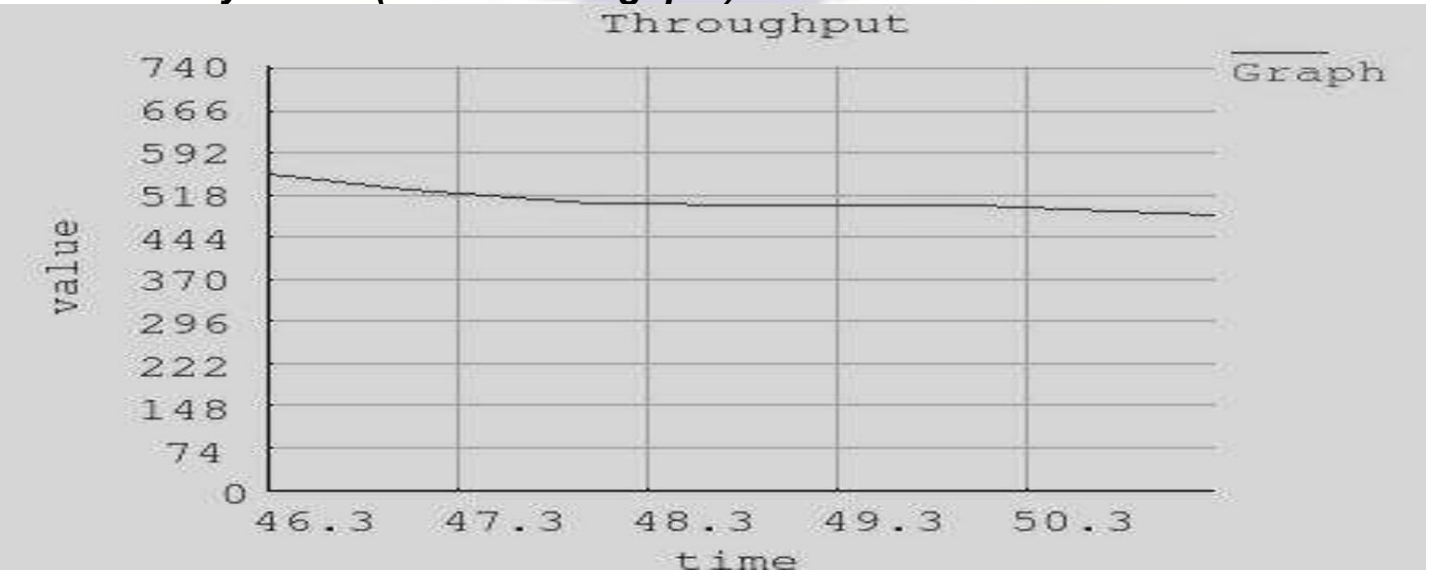

Fig. 8. Signal attenuated by $2 \mathrm{dbm}$ (Throughput) 
The above graph show the throughput when the signal strength is attenuated by $2 \mathrm{dbm}$ after the communication is out of obstacles. As mentioned before, the communication continues as normal for 24 seconds. At 46.3 seconds, the node reached $2 \mathrm{dbm}$ signal attenuator during communication. At this time, the signal strength is attenuated by $2 \mathrm{dbm}$ (free medium). This situation occurs within 7 seconds. As a result shown in the above graph (Figure 4.8), starting from 46.3 seconds, the number of throughput is decreased from about 555 bps to 481 bps.

\section{Signal attenuated by $3 \mathrm{dbm}$ (Metric Dropped Packet)}

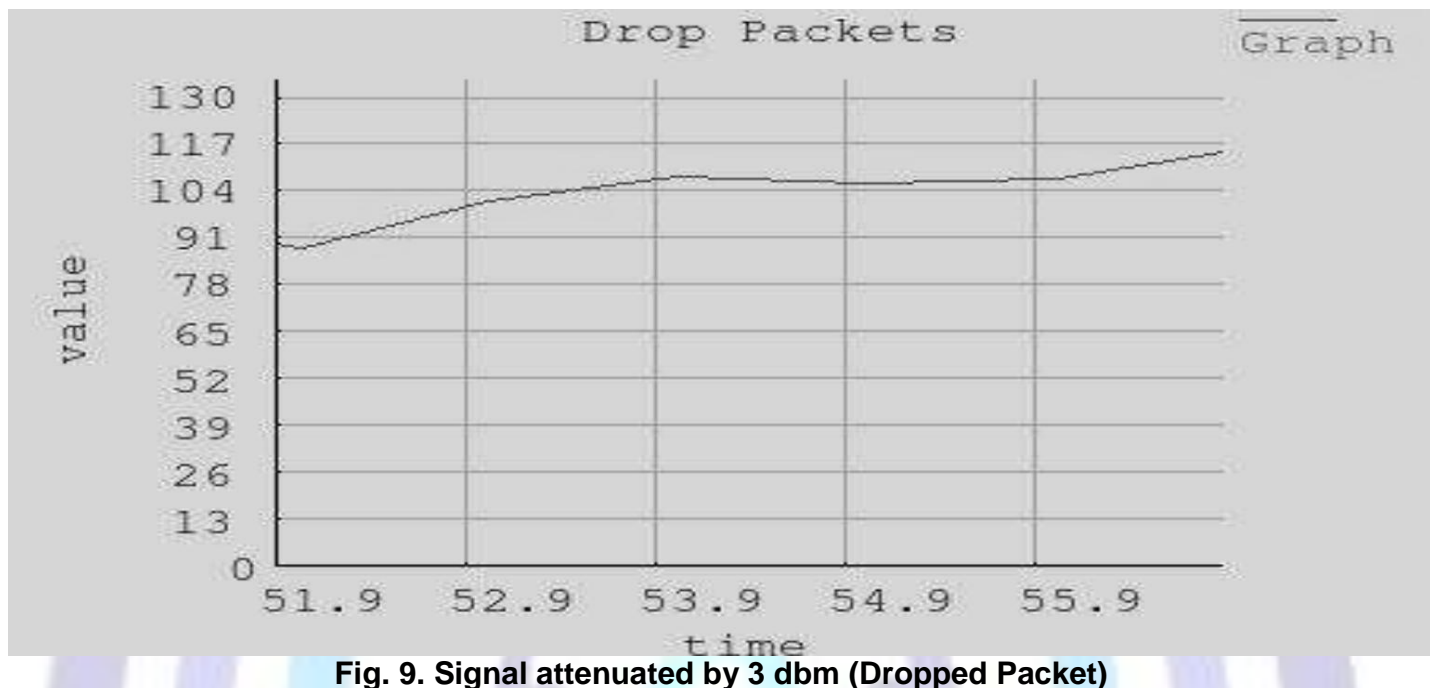

Fig. 9. Signal attenuated by $3 \mathrm{dbm}$ (Dropped Packet)

After 7 seconds the signal had been attenuated by $2 \mathrm{dbm}$, the node reached the second signal attenuator. At 51.9 seconds, the node reached $3 \mathrm{dbm}$ signal attenuator during communication. At this time, the signal strength is attenuated by $3 \mathrm{dbm}$ (free medium). This situation occurs within 5 seconds.

The above graph show the dropped packet when the signal strength is attenuated by $3 \mathrm{dbm}$. As a result shown in the above graph (Fig. 9), starting from 51.9 seconds, the number of dropped packets is increased from about 91 packet/s to almost 117 packet/s.

\section{Signal attenuated by $3 \mathrm{dbm}$ (Metric Throughput)}

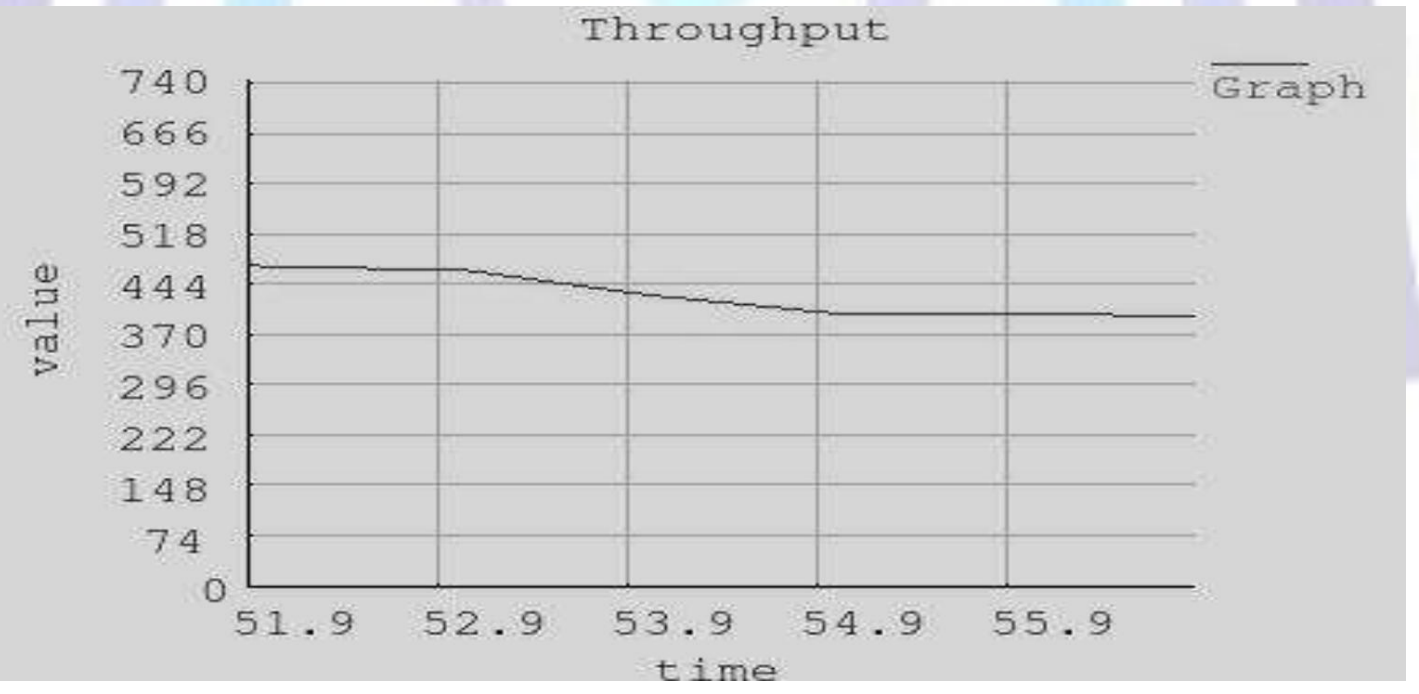

Fig. 10. Signal attenuated by $3 \mathrm{dbm}$ (Throughput)

After 7 seconds the signal had been attenuated by $2 \mathrm{dbm}$, the node reached the second signal attenuator. At 51.9 seconds, the node reached $3 \mathrm{dbm}$ signal attenuator during communication. At this time, the signal strength is attenuated by $3 \mathrm{dbm}$ (free medium). This situation occurs within 5 seconds.

The above graph show the throughput when the signal strength is attenuated by $3 \mathrm{dbm}$. As a result shown in the above graph (Fig. 10), starting from 51.9 seconds, the number of dropped packets is decreased from about 481 bps to almost 404 bps.

\section{Comparative Study}




\section{AODV Performance Using OPNET}

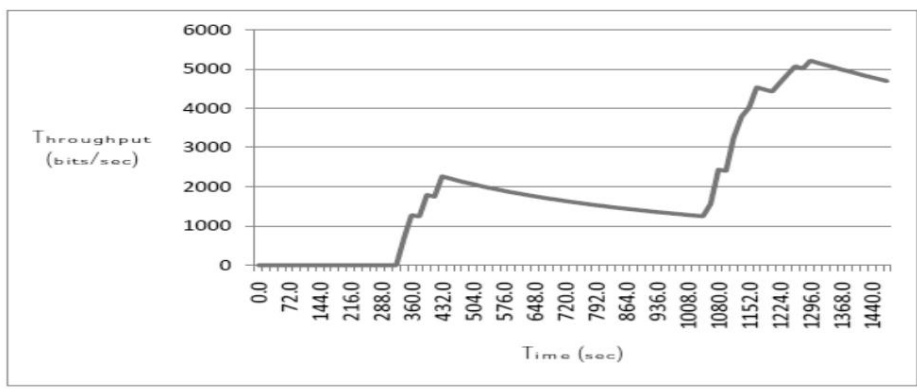

Fig. 11. Throughput of AODV

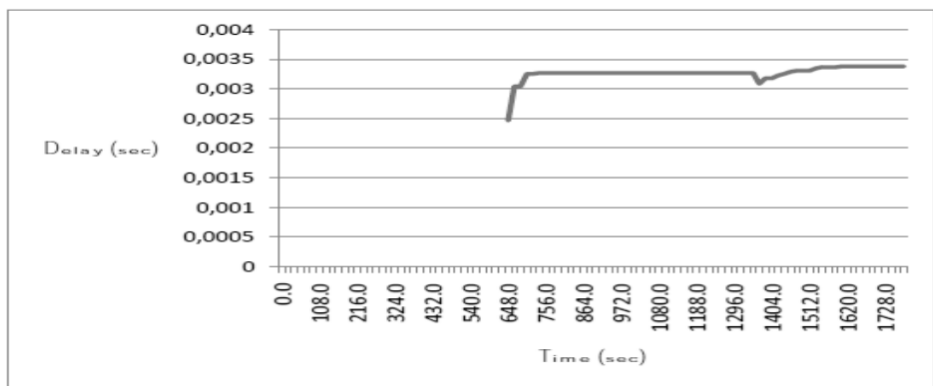

Fig. 12. End Delay of AODV

Asar Ali and Zeeshan Akbar [21] obtained the result for the performance of the routing protocols measured by throughput and end-to-end delay metrics as shown in the both Figures 4.11 and 4.12 .

The simulation was setup based on the following scenario:

- Number of Nodes $=10$ nodes

- Types of Nodes = Mobile

- Mobility Model = FTP

- Mobility Speed $=10 \mathrm{~m} / \mathrm{s}$

- Packet Size $=512$ byte

- Topology Size $=500 \mathrm{~m} \times 500 \mathrm{~m}$

- Time $=1800$ seconds

From the Fig. 11, in the time range from 648 to 1080 seconds, the throughput is decreased from almost 2000 bps to almost 1000 bps. Inversely, from the Figure to 4.12 , even the end-to-end delay is static but it is not decreased, and beside that it increased after 1404 seconds while the throughput is decreased. Based on that, we can say when the throughput increased, the dropped packet decreased and inversely.

AODV Performance Using NS-2 (Case-1)

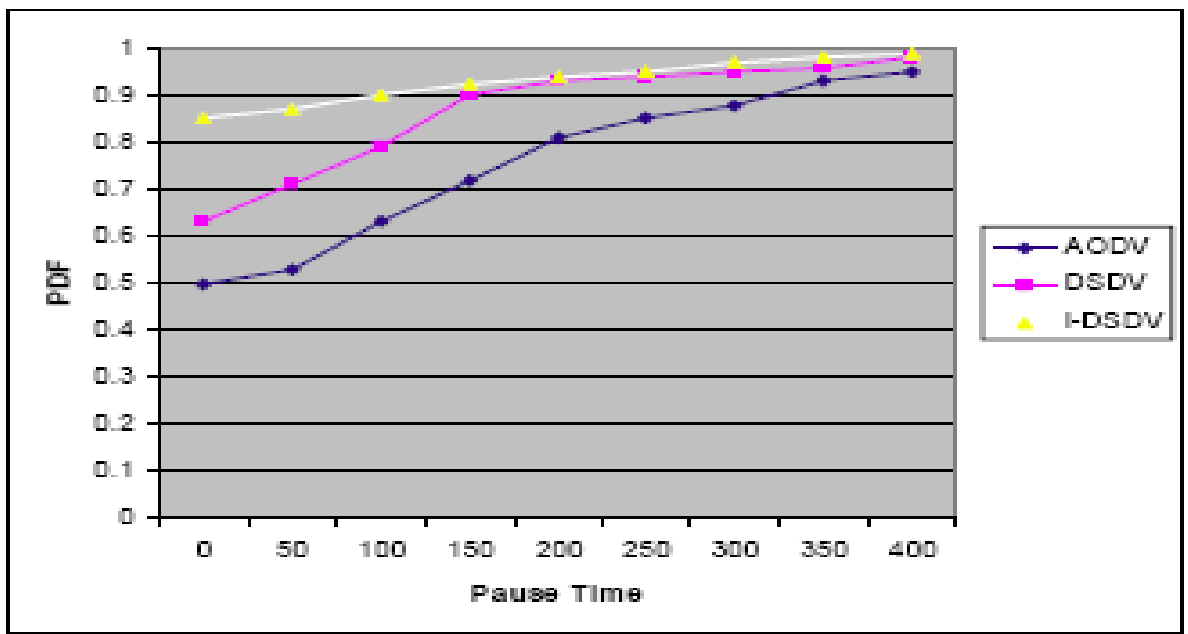


Fig. 13. Packet Delivery Fraction (Case-1)

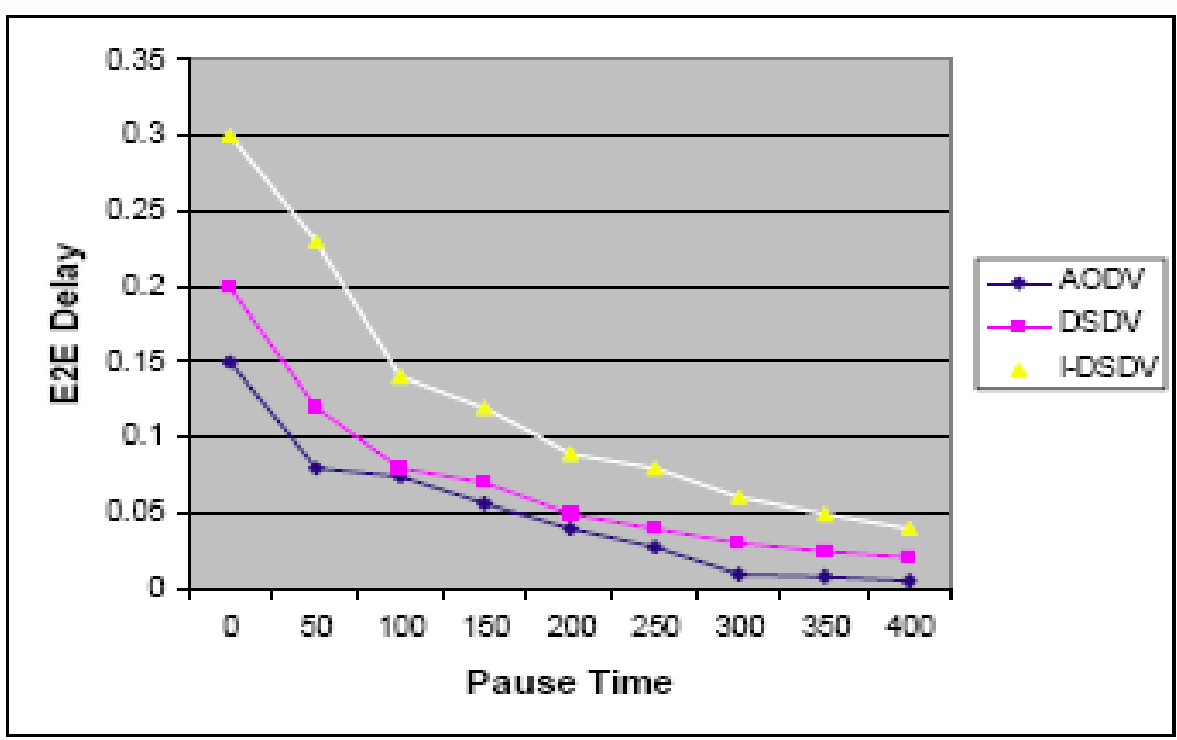

Fig. 14. End-To-End Delay (Case-1)

Abdul Hadi Abd Rahman and Zuriati Ahmad Zulkarnain [23] obtained the above two graphs. The simulation is conducted to evaluate the routing protocols in different pause time while the number of nodes and the node speed are fixed. The node speed is set to $20 \mathrm{~m} / \mathrm{s}$ and the number of nodes is set to 20 nodes. The pause time are set to $0,50,100,150,200$, 250, 300, 350 and 400 second. Based on Fig. 13, we can see clearly that the Packet delivery Fraction or throughput is increased from $0.50 \mathrm{kbps}$ into $0.95 \mathrm{kbps}$ during the pause time from 0 to 400 seconds. Based on Fig. 14, it shows that end-to-end delay is decreased from 0.15 packet/s into almost 0.01 packet/s. So, we can concluded that when the throughput increased, the dropped packet decreased.

\section{AODV Performance Using NS-2 (Case-2)}

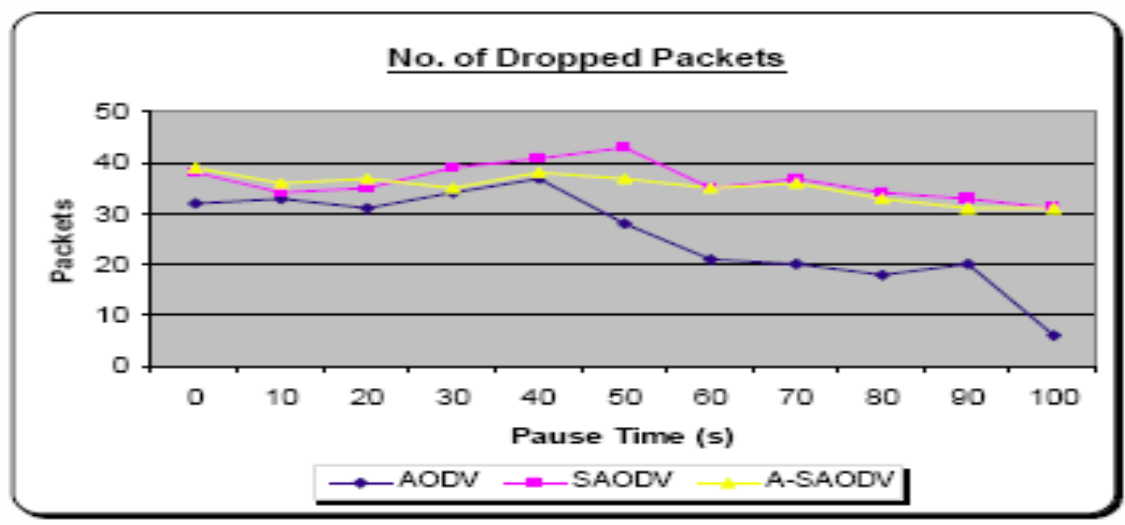

Fig. 15. No. of Dropped Packet 


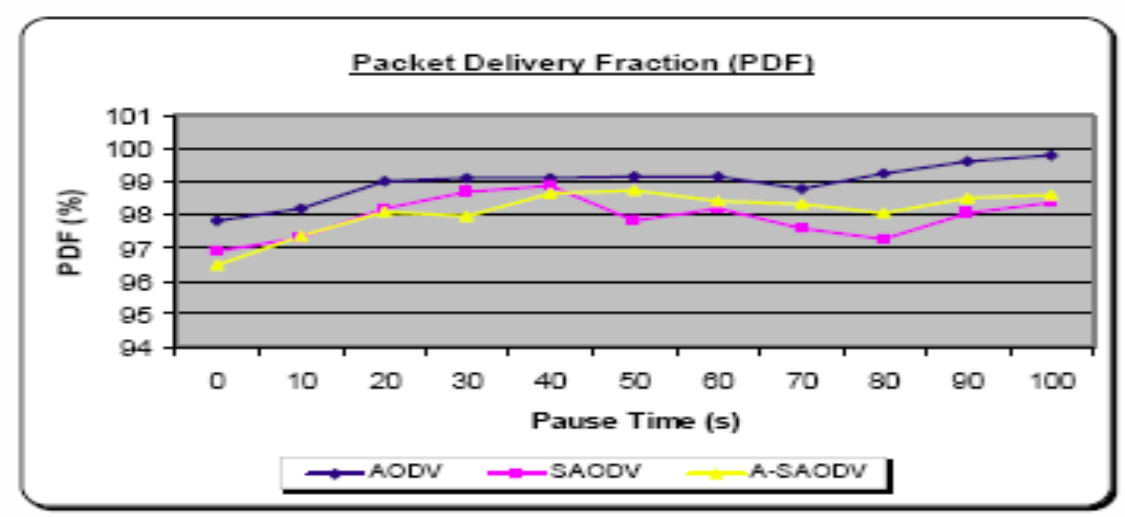

Fig. 16. Packet Delivery Fraction (Case-2)

Mohd Anuar Jaafar and Zuriati Ahmad Zulkarnain [24] had obtained the above two graphs about the performance of AODV. The scenario and the environment settings are fixed. It is purposely done to see the performance of AODV routing protocol in a chosen free-attack simulation environment using throughput and PDF performance metrics.

The simulation was setup based on the following scenario:

- Number of Nodes $=50$ nodes

- Maximum connections $=40$ traffic sources

- Mobility Model = Random Waypoint

- Mobility Speed $=40 \mathrm{~m} / \mathrm{s}$

- Rate $=8 \mathrm{kbps}(2$ packets per load $)$

- Topology Size $=500 \mathrm{~m} \times 500 \mathrm{~m}$

- Time $=100$ seconds (results are collected every 10 s of pause time)

Based on Fig. 15, it shows the total number of dropped packets that have been collected during the running of the simulations. AODV had the lowest number of dropped packets compared to the other routing protocols and became nearly 0 dropped packets produced, as it moves to a larger pause time. At larger pause time, AODV having smaller quantities of dropped packets. Based on Fig. 16, PDF shows how successful a protocol performs delivering packets from source to destination. Higher value (nearest to 1.0) means the better the results. The throughput is increased from 98 to $100 \mathrm{kbps}$ from time 0 to time 100. So from the both graphs, it means that when the throughput increased, the dropped packet decreased and inversely.

\section{AODV Performance Using NS-2 (Case-3)}

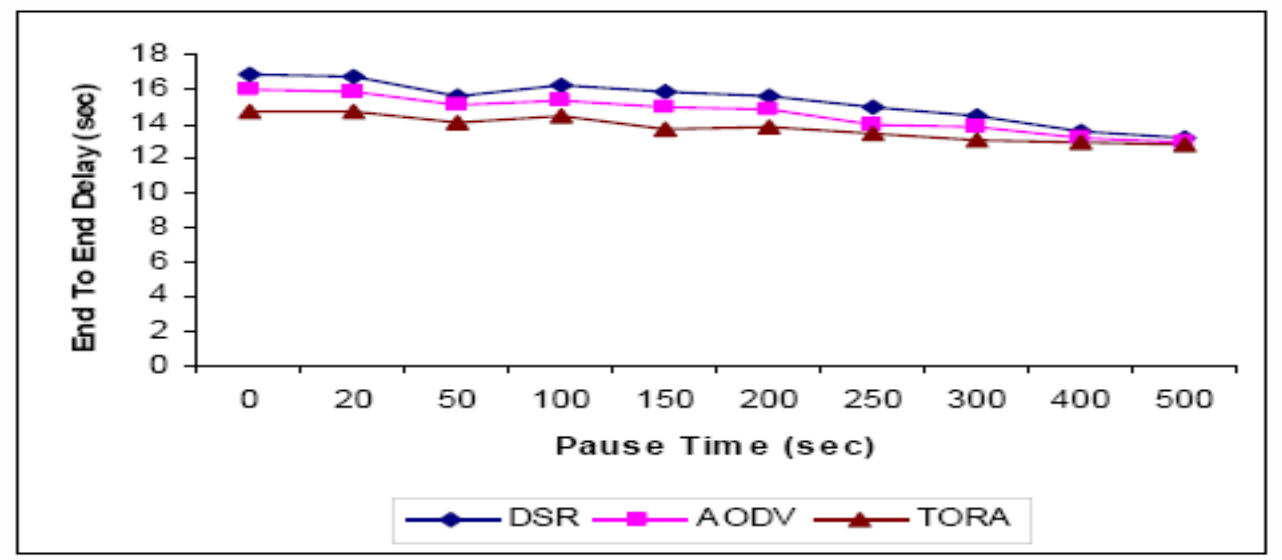

Fig. 17. End-To-Énd Delay (Case-2) 


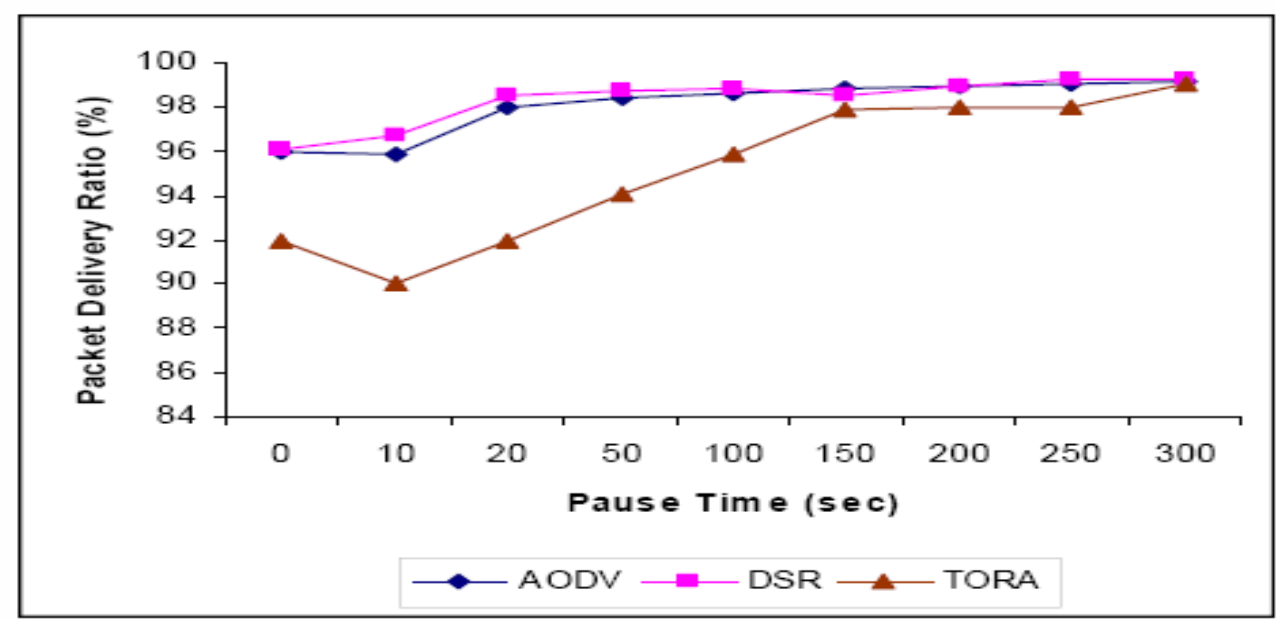

Fig. 18. Packet Delivery Fraction (Case-3)

Anuj K. Gupta [25] had obtained the above two graphs about the performance of AODV. The scenario and the environment settings are fixed. It is purposely done to see the performance of AODV routing protocol in a chosen freeattack simulation environment using throughput and PDF performance metrics.

The simulation was setup based on the following scenario:

- Transmission Range $=250 \mathrm{~m}$

- Traffic Type = CBR

- Mobility Model = Random Waypoint

- Bandwidth = $2 \mathrm{Mbps}$

- Topology Size $=500 \mathrm{~m} \times 500 \mathrm{~m}$

- Simulation Time $=200$ seconds

Based on Fig. 17, it shows the total number of end-to-end delay that have been collected during the running of the simulations. The number of end-to-end delay for AODV is decreased and became smaller to a larger pause time. At larger pause time, AODV having the value of end-to-end delay about 12 seconds. Based on Fig. 18, PDF shows how successful a protocol performs delivering packets from source to destination. Higher value (nearest to 98) means the better the results. The throughput is increased from 96 to almost $100 \mathrm{kbps}$ from time 0 to time 300 . So from the both graphs, it means that when the throughput increased, the dropped packet decreased and inversely.

\section{CONCLUSION AND DISCUSSION}

This chapter consists of three sections. First, the conclusion from simulation study of AODV for VANET. The second section is the conclusion from comparative study of AODV taken from any articles and journals. The last section is an overall discussion about the performance of AODV routing protocol.

\section{Conclusion from Simulation Study}

The practical implementation through VANET simulation was performed in this dissertation. In simulation, an obstacle or hindering object and signal attenuator with different $\mathrm{dbm}$ had been inserted in the communication medium. Two parameters were used in order to evaluate the performance of the VANET, i.e. throughput and dropped packet.

From all the graphs produced by the simulation process, we can conclude that the performance of AODV is affected by hindering objects and signal strength. We can conclude that the throughput is very low by using multi-hop communication. The number of dropped packets increases as the signal strength increased. Then based on the result, we can conclude that the throughput is greatly affected by hindering objects and the number of dropped packets is affected more by signal attenuation.

As the conclusion from simulation study for VANET simulation using AODV routing protocol, we can conclude that when the throughput is increased, the dropped packet will be decreased and vice versa.

\section{Conclusion from Comparative Analysis}

Based on the comparative analysis, as far as we concerned, we can conclude that the performance of AODV is independent from the type simulator used in simulation. This statement had been approved from the comparative analysis obtained from the previous section whereas the result from a few articles and journals were obtained from the different simulators i.e. NCTUns, OPNET and NS-2.

As the conclusion from comparative analysis for VANET simulation using AODV routing protocol, we can conclude that when the throughput is increased, the dropped packet will be decreased and vice versa.

\section{Discussion}

In this paper, we have evaluated the performance of AODV routing protocols under hindering object or obstacle and the changes of signal strength using NCTUns version 5.0 network simulator with Raleigh fading model. The protocol 
represents the proactive routing protocols. The performance was evaluated by measuring packet delivery fraction or throughput and dropped packet.

We had also evaluated the performance of AODV routing protocol using different network simulator by doing comparative analysis from literature review. The performance was evaluated and compared by measuring throughput and end-to-end delay (equivalent to dropped packet) from the simulation results.

As an overall conclusion, based on the above simulation and comparative study, we can conclude that:

1. The performance of AODV routing protocol is affected by hindering objects and by the strength of signal in VANET simulation.

2. The rate of the throughput and the dropped packet of AODV routing protocol for VANET is opposite each other. In other word, when the throughput increased, the dropped packet is increased and vice versa.

The same result obtained due to the relationship between throughput and dropped packet when different type of simulator is used. So we can conclude that the performance of AODV is independent from type of network simulator.

\section{REFERENCES}

[1] Kevin C. Lee, Uichin Lee, and Mario Gerla, "Survey of Routing Protocols in Vehicular Ad Hoc Networks", Computer Science Department, University of California, Los Angeles, 2008.

[2] Shie-Yuan Wang and Chih-Liang Chou, "NCTUns Simulator for Wireless Vehicular Ad Hoc Network Research", Department of Computer Science, National Chiao Tung University, Hsinchu, Taiwan, 2008.

[3] H.P. Glathe, L. Karlsson, G.P. Brusaglino, L. Calandrino, "The PROMETHEUS Programme - Objectives, Concepts and Technology for Future Road Traffic", XXIII FISITA Congress, May 1990, pp. 477-484.

[4] C. Sommer, I. Dietrich, F. Dressler, "Realistic Simulation of Network Protocols in VANET Scenarios", Mobile Networking for Vehicular Environments 2007 Volume, Issue, 11-11 May 2007, pp.139-143.

[5] Jerome Haerri, "Performance Comparison of AODV and OSLR in VANETs Urban Environments under Realistic Mobility Patterns", Department of Mobile Communication B.P. 193 06904, Sophia Antipolis, France.

[6] N.C. Romano and J.F. Numamaker, "Meeting Analysis: Findings from Research and Practice", in Proceedings of The $34^{\text {th }}$ Hawaii International Conference on System Science, 2001.

[7] Shie-Yuan Wang, Chih-Che Lin, "NCTUns 5.0: A Network Simulator for IEEE 802.11(p) and 1609 Wireless Vehicular Network", Department of Computer Science, National Chiao Tung University, Hsinchu, Taiwan.

[8] M. Fiore, J. Harri, F. Filali, C. Bonnet, "Vehicular Mobility Simulation for VANETs Simulation", Symposium, 2007. ANSS apos;07. 40 ${ }^{\text {th }}$ Annual Volume, Issue, 26-28 March 2007, pp.301-309.

[9] Cristian Tuduce and Thomas Gross, "A Mobility Model Based on WLAN Traces and its Validation", in Proceedings of the IEEE INFOCOM 2005, Miami, March 2005.

[10] Rajive Bagrodia, Richard Meyer, Mineo Takai, Yu An Chen, Xiang Zeng, Jay Martin, and Ha Yoon Song, "Parsec: $A$ Parallel Simulation Environment for Complex Systems Computer", 1998, pp. 77-85.

[11] Tony Larson and Nicklas Hedman, "Routing Protocols in Wireless Ad Hoc Network - A Simulation Study", Department of Computer Science and Electrical Engineering, Lulea University of Technology, Stockholm, 1998.

[12] Lee U., Zhou B., Gerla M., Magistretti E., Bellavista P. and Corradi A., "Mobeyes: Smart Mobs for Urban Monitoring with A Vehicular Sensor Network”, Wireless Communication, IEEE, vol.13, no. 5, pp.52-57, October 2006.

[13] Pei G., Gerla M., and Chen T., "Fisheye State Routing: A Routing Scheme for Ad Hoc Wireless Networks", Proc. ICC 2000, New Orleans, June 2000.

[14] Jungkeun Yoon, Brian D. Noble, Mingyan Liu, Minkyong Kim, "Building Realistic Mobility Models from Coarsegrained Traces", in Proceedings of the ACM International Conference On Mobile Systems, Applications and Services (MobiSys'06), pp. 177-190, 2006.

[15] Flury R. and Wattenhofer R., "MLS: An Efficient Location Service for Mobile Ad Hoc Networks", in MOiHoc '06: proceedings of the $7^{\text {th }}$ ACM International Symposium on Mobile Ad Hoc Networking and Computing, pp.226-237, New York, USA, 2006.

[16] Perkins C.E. and Royer E.M., "Ad Hoc on Demand Distance Vector Routing", in Proc. IEEE WMCSA '99, New Orleans, LA, Feb. 1999, pp.90-100.

[17] Park V.D. and Corson M.S., "A Highly Adaptive Distributed Routing for Mobile Wireless Networks", INFOCOM 1997. Sixteenth Annual Joint Conference of the IEEE Computer and Communication Societies. Proceedings IEEE, vol.3, no., pp. 1405-1413, April 1997.

[18] Jaap S., Bechler M., and Wolf L., "Evaluation of Routing Protocols for Vehicular Ad Hoc Networks in City Traffic Scenarios", Proceedings of the $5^{\text {th }}$ International Conference on Intelligent Transportation System (ITS) Telecommunications, June, 2005.

[19] Lochert C., Hartenstein H., Tian J., Fussler H., Hermann D. and Mauve M., "A Routing Strategy for Vehicular Ad Hoc Networks in City Environments", Intelligent Vehicles Symposium, 2003. Proceedings IEEE, vol., no., pp.156161, June 2003.

[20] Zhao J., and Cao G., "VADD: Vehicle-Assisted Data Delivery in Vehicular Ad Hoc Networks", INFOCOM 2006. 25" IEEE International Conference on Computer Communication. Proceedings, vol., no., pp. 1-12, April 2006.

[21] Asar Ali and Zeeshan Akbar, "Evaluation of AODV and DSR Routing Protocols of Wireless Sensor Networks for Monitoring Applications", Electrical Engineering and Telecommunication, Kalskrona, 2009.

[22] Imran Khan and Amir Qayyum, "Performance Evaluation of AODV and OLSR in Highly Fading Vehicular Ad hoc Network Environments", Center of Research in Networks and Telecom (CoReNeT), M. A. Jinnah University, Jinnah Avenue, Blue Area, 44000, Islamabad, Pakistan, 1984. 
[23] Abdul Hadi Abdul Rahman and Zuriati Ahmad Zulkarnai, "Performance Comparison of AODV, DSDV and I-DSDV Routing Protocols in Mobile Ad Hoc Networks", European Journal of Scientific Research, ISSN 1450-216X Vol.31 No.4 (2009), pp.566-576, (C) EuroJournals Publishing, Inc. 2009, http://www.eurojournals.com/ejsr.htm

[24] Mohd Anuar Jaafar and Zuriati Ahmad Zulkarnain, "Performance Comparisons of AODV, Secure AODV and Adaptive Secure AODV Routing Protocols in Free Attack Simulation Environment", European Journal of Scientific Research, ISSN 1450-216X Vol.32 No.3 (2009), pp.430-443 @ EuroJournals Publishing, Inc. 2009, http://www.eurojournals.com/ejsr.htm

[25] Anuj K. Gupta, "Performance analysis of AODV, DSR \& TORA Routing Protocols", IACSIT International Journal of Engineering and Technology, Vol.2, No.2, April 2010, ISSN: 1793-8236.

[26] Naumov, V. Gross and T.R.,"Connectivity-Aware Routing (CAR) in Vehicular Ad-Hoc Network", INFOCOM, 26 IEEE International Conference on Computer Communication, pp. 1919-1927, 6-12 May, 2007.

\section{Author's Biography}

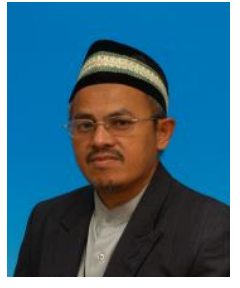

Mr. Mazlan Osman had been working as a lecturer at Universiti Teknologi MARA (UiTM) in Malaysia. He had been working at UiTM almost fifteen years and he had Master Degree in Computer Networking. His research areas are Data Communication, Wireless Network and Network Security. He is also the member of Network Profesional Association (NPA).

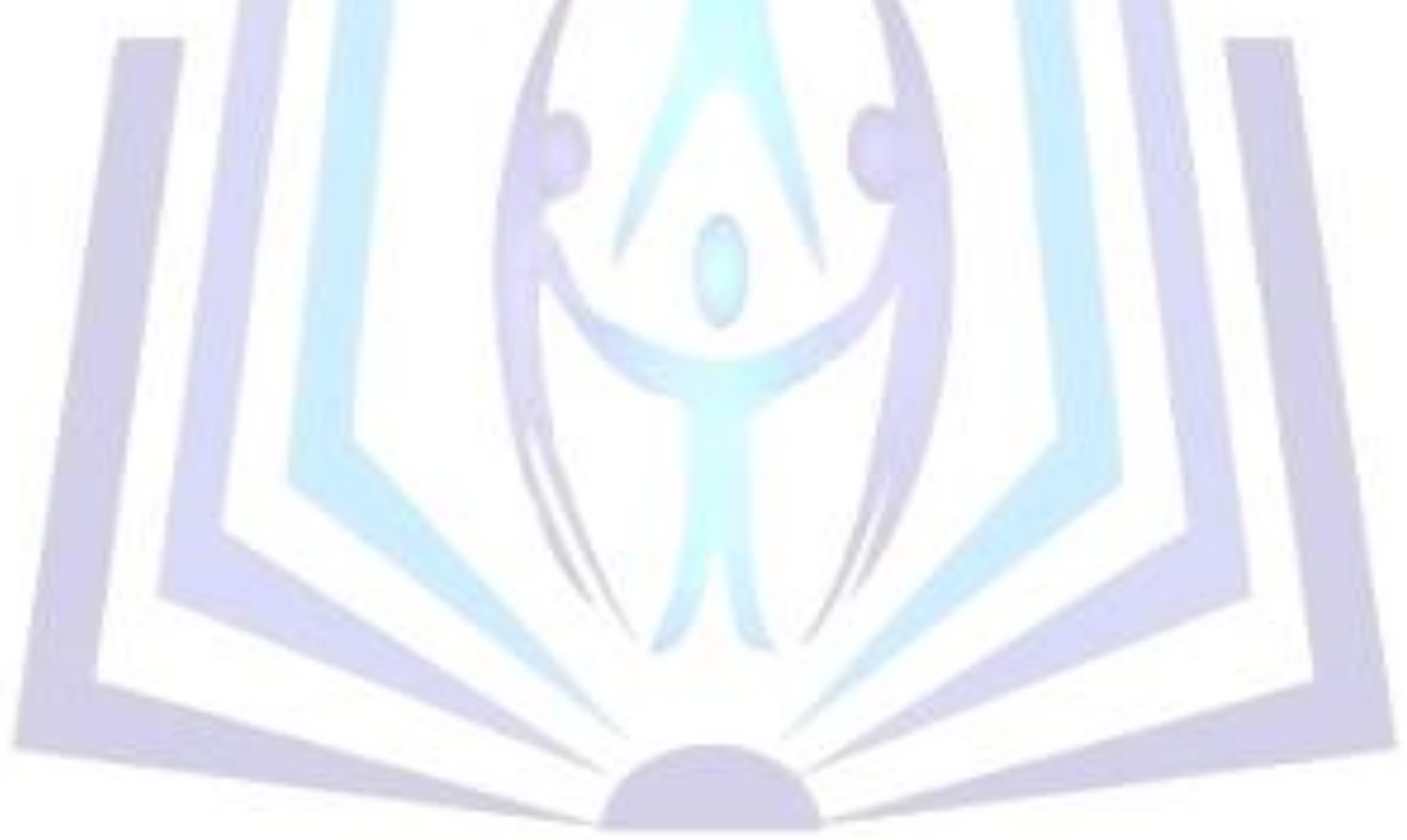

